\title{
Port-Hamiltonian Mathematical Model of a Fluid Ring Attitude System
}

\author{
Juan Cristobal Alcaraz Tapia ${ }^{+}(\mathbb{D})$, Carlos E. Castañeda ${ }^{*}+\mathbb{D}^{(\mathbb{D})}$ and Héctor Vargas-Rodríguez ${ }^{+}(\mathbb{D})$ \\ Ciencias Exactas y Tecnología, Centro Universitario de los Lagos, Universidad de Guadalajara, \\ Lagos de Moreno 47460, Jalisco, Mexico; cristobal.alcaraz@alumnos.udg.mx (J.C.A.T.); \\ hvargas@culagos.udg.mx (H.V.-R.) \\ * Correspondence: ccastaneda@lagos.udg.mx or carlose.castanedah@academicos.udg.mx \\ t These authors contributed equally to this work.
}

Citation: Alcaraz Tapia, J.C.;

Castañeda, C.E.; Vargas-Rodríguez, H. Port-Hamiltonian Mathematical Model of a Fluid Ring Attitude System. Energies 2021, 14, 6906 https://doi.org/10.3390/en14216906

Academic Editors: Robert Castilla and Dmitry Eskin

Received: 17 August 2021

Accepted: 14 October 2021

Published: 21 October 2021

Publisher's Note: MDPI stays neutral with regard to jurisdictional claims in published maps and institutional affiliations.

Copyright: (c) 2021 by the authors. Licensee MDPI, Basel, Switzerland. This article is an open access article distributed under the terms and conditions of the Creative Commons Attribution (CC BY) license (https:/ / creativecommons.org/licenses/by/ $4.0 /)$.

\begin{abstract}
In this article, we propose a mathematical model using the port-Hamiltonian formalism for a satellite's three-axis attitude system comprising fluid rings. Fluid rings are an alternative to reaction wheels used for the same purpose, since, for the same mass, they can exert a greater torque than a reaction wheel as the fluid can circulate the periphery of the satellite. The port-Hamiltonian representation lays the foundation for a posterior controller that is feasible, stable, and robust based on the interconnection of the system to energy shaping and/or damping injection components, and by adding energy routing controllers. The torques exerted by the fluid rings are modeled using linear regression analysis on the experimental data got from a prototype of a fluid ring. Since the dynamics of turbulent flows is complex, the torques obtained by the prototype lead to a simpler first approach, leaving its uncertainties to a controller. Thus, the attitude system model could be tested in a future prototype before considering a spatial environment.
\end{abstract}

Keywords: Euler's equations; attitude system; fluid rings; satellite; port-Hamiltonian system

\section{Introduction}

For the operation of an artificial satellite, it must be oriented with respect to other celestial bodies. For this, it is necessary to use devices that produce a torque on the satellite, and that continuously correct its attitude (spatial orientation). These devices are part of the attitude control system. The literature describes various attitude control systems integrated by different momentum exchange devices (torque generating devices). Among the latter, reaction wheels have a relatively simple construction and have been widely studied and used for a long time [1-6]. Reaction wheels allow for precise changes to be made in the attitude of the satellite and do not require the ejection of mass unlike the systems incorporating thrusters. Control momentum gyroscopes (CMG's) are more complex than the reaction wheels but, to exert a given torque onto a spacecraft, CMG's typically require less energy than reaction wheels. For the $\mathrm{CMG}^{\prime}$ 's there is also a great quantity of information about law designs or problems with singular gimble angle configurations [7-10]. Some devices use the Earth's magnetic field to achieve attitude corrections [11-13], therefore, magnetic torquing is a good option for small satellites in low Earth orbits. These kinds of systems are lightweight, inexpensive, and require low power, but, as a limitation, the torque exerted is perpendicular to the local geomagnetic field vector.

Another momentum exchange device [14] uses the motion of a fluid in a circuit to exert a torque onto a spacecraft. In the literature, a round-shaped circuit is often used and it is usually called fluid ring. Since the mass of a fluid ring is concentrated in the periphery, it can exert a greater torque onto a satellite than a reaction wheel of similar mass. This idea has led to further investigation of this type of device. Varatharajoo in 2003 [15] suggested the coupling of existing spacecraft subsystems to decrease the system volume/mass and complexity. The proposed concept considers electrically conducting fluids in a closed-loop 
for the attitude and thermal control systems, employing an electromagnetic pump. In the work of Xiao [16], it is considered that the permanent magnets mentioned by Varatharajoo might influence the sensitive equipment, influencing the normal work of the satellite. Besides, the torque generated was small. Therefore, Xiao proposes the use of a mechanical pump as well as a mathematical model for a small satellite controller based on mechanicallypumped fluid loops. Due to the similarity to the controllers in three axes, Xiao built the mathematical model of one axis, considering a fluid ring, the mechanical pump, and the motor connected to it. The simulations proved the effectiveness and feasibility of its application since there were improvements in performance and volume/mass savings compared to reaction wheels. In 2004 Kelly [17], also proved the feasibility of using fluid rings by testing the torque exerted by rings filled with water onto a prototype aboard the KC-135A. In 2009 Kumar [18] made a more complex model for a configuration of three fluid rings considering the effect of the gravity gradient torque on the fluid ring controlled satellites, stability analysis, and the effectiveness of fluid rings in controlling the satellite attitude in presence of attitude disturbances and actuators' failures. Nobari and Misra [19] pointed out that the dynamical model developed by Kumar does not include all reaction moments transferred between the satellite and the fluid rings; furthermore, they improved Kumar's model by developing a complete version, using four fluid rings in a pyramidal configuration. This type of attitude controller has also been proposed as a part of a hybrid attitude system consisting of two magnetic rods and a fluid ring [20]. Also, there are comparatives of CMG and fluid rings [21,22], concluding that CMGs are superior in large torque amplification and momentum storage capacity when compared to the fluid rings for agility maneuvers, but the mechanism of the fluid rings is simpler when comparing to CMGs. The latter works have in common the representation of two nonlinear coupled ordinary differential equations for the angular momentum of the satellite and the fluid rings, respectively, where the torque exerted by the system of fluid rings appears, but not explicitly the torque exerted by each fluid ring in the direction of its symmetry axis. Furthermore, these torques involve fluid mechanics which are complex to model.

Regarding nonlinear models, the port-Hamiltonian paradigm offers powerful tools for analysis, control, and simulation of physical systems. As this type of modeling puts emphasis on energy and power, it offers a framework for modeling systems of different domains (mechanical, electrical, hydraulic, etc.). Furthermore, it allows for exploitation of the physical properties of a system such as balance and conservation laws. Thus, the portHamiltonian modeling has brought considerable attention and has been building a solid foundation concerning analysis and control [23-31]. The aforementioned results motivate us to propose a Port-Hamiltonian mathematical representation of a fluid ring attitude system for an artificial satellite. The fluid rings can exert greater torques than reaction wheels, and the port-Hamiltonian representation allows the development of more complex systems from the interconnection of simpler parts with the goal of proposing robust control algorithms in future works. Also, an attitude model described by Euler's equations can be written as a port-Hamiltonian system, with both having the same behavior.

In this work, a mathematical model for the attitude system of an artificial satellite is proposed, comprising three fluid rings. The attitude is represented by the (3-2-1) set of Euler angles and the dynamics of the satellite is described as a port-Hamiltonian system where the canonical variables are generalized coordinates and momenta. The time derivatives of the generalized momenta are obtained by using Euler's rotational equations of motion. As a simple example, the torques produced by the pump in each fluid ring are obtained experimentally, using linear regression analysis on the data collected from a fluid ring prototype, relating the volume flow rate and the electric current supplied to the pump. Consequently, a simpler representation for the torques is obtained, instead of involving the complexity of turbulent flow dynamics.

The main contribution of this work is to obtain a port-Hamiltonian mathematical representation of a fluid ring attitude system considering a linear regression model for the torques produced by the fluid rings. 
This work is organized as follows: Section 2 describes the set of Euler angles chosen as the attitude coordinates. Then, a dynamical model using Euler's equations of motion is developed. Lastly, from the Euler's equations and the Hamiltonian of the system, a model is written in the port-Hamilton form; Section 3 shows a comparison of the open-loop simulation between the port-Hamitonian system and Euler's equations of motion, showing that both representations describe the same dynamics. Also, the model of the torques obtained experimentally using linear regression analysis on the data collected from a fluid ring prototype is collected; the conclusions of the work are presented in Section 4; and finally, the inertia matrix of the fluid rings is presented in Appendix A.

\section{Materials and Methods}

This section is divided into three parts; the first one introduces the attitude parameters that will be used to describe the orientation of the satellite. Then, its dynamical model is written using Euler's equations of motion. Lastly, the model is represented in a portHamiltonian form.

\subsection{Euler Angles (Yaw, Pitch and Roll)}

In celestial mechanics, as well as in the study of rigid body dynamics, Euler angles are frequently used to relate two coordinate systems [32]. Aircraft and spacecraft orientations are commonly described through the Euler angles: yaw $(\psi)$, pitch $(\theta)$, and roll $(\phi)$ measured in radians. The transformation of a vector in an inertial frame $(\mathcal{N})$ into a reference frame (e.g., one fixed to a body, in this case, named $\mathcal{B}$ ), defined by the unit vectors $\hat{\boldsymbol{b}}_{1}, \hat{\boldsymbol{b}}_{2}$, and $\hat{\boldsymbol{b}}_{3}$, is realized through a sequence of Euler angle rotations. The reference axes are first rotated about the $\hat{\boldsymbol{b}}_{3}$ axis by the yaw angle $\psi$, then about the $\hat{\boldsymbol{b}}_{2}$ axis by the pitch angle $\theta$, and finally about the $\hat{\boldsymbol{b}}_{1}$ axis by the roll angle $\phi$. Thus, the standard yaw-pitch-roll $(\psi, \theta, \phi)$ angles are the (3-2-1) set of Euler angles [33]. For a fixed frame $\mathcal{B}$ orbiting around another body at an angular velocity $\Omega$, the kinematic differential equations can be written as in the following subsection.

\section{Kinematic Differential Equations}

The relation between the angular velocities in a body frame $\mathcal{B}$ and the Euler rates $(\dot{\psi}, \dot{\theta}, \dot{\phi})$ using the (3-2-1) set of Euler angles is [33,34]:

$$
\left[\begin{array}{c}
\dot{\psi} \\
\dot{\theta} \\
\dot{\phi}
\end{array}\right]=[\mathbf{B}(\psi, \theta, \phi)]^{\mathcal{B}} \dot{\boldsymbol{q}}_{s}-\frac{\Omega}{\cos \theta}\left[\begin{array}{c}
\sin \theta \sin \psi \\
\cos \theta \cos \psi \\
\sin \psi
\end{array}\right]
$$

where the matrix $[\mathbf{B}(\psi, \theta, \phi)]$ is

$$
[\mathbf{B}(\psi, \theta, \phi)]=\frac{1}{\cos \theta}\left[\begin{array}{ccc}
0 & \sin \phi & \cos \phi \\
0 & \cos \phi \cos \theta & -\sin \phi \cos \theta \\
\cos \theta & \sin \phi \sin \theta & \cos \phi \sin \theta
\end{array}\right]
$$

and the vector ${ }^{\mathcal{B}} \dot{\boldsymbol{q}}_{s}=\left[\begin{array}{lll}q_{s 1} & q_{s 2} & q_{s 3}\end{array}\right](\mathrm{rad} / \mathrm{s})$ represents the angular velocity of the $\mathcal{B}$ frame respect to the $\mathcal{N}$ frame in terms of the $\mathcal{B}$ frame components $\left(\hat{\boldsymbol{b}}_{1}, \hat{\boldsymbol{b}}_{2}, \hat{\boldsymbol{b}}_{3}\right)$.

The angular velocity $\Omega$ is given by Kepler's equation:

$$
\Omega^{2}=\frac{G M_{e}}{R_{c}^{3}}
$$

where $G$ is the universal gravitational constant, $M_{e}$ is Earth's mass, and $R_{c}$ is the magnitude of an inertial position vector measured from Earth's center.

Equation (1) shows the Euler rates as a function of the Euler angles, the angular velocity of the $\mathcal{B}$ frame, and $\Omega$, and it is a subsystem of the complete model that describes the dynamics of a satellite's attitude system. 


\subsection{Dynamical Modeling}

The proposed attitude system concerning three fluid rings can be seen in Figure 1, where the satellite is represented as a cube with a fluid ring on each of its three visible faces. Each ring's symmetry axis coincides with a satellite's principal axis $(X, Y$, and $Z)$. The $\mathcal{B}$ frame is fixed to the satellite, and the unit vectors $\hat{\boldsymbol{b}}_{1}, \hat{\boldsymbol{b}}_{2}, \hat{\boldsymbol{b}}_{3}$ are aligned with the satellite's principal axes. The matrices of inertia of the satellite and the fluid rings are presented in Section 2.2.1, and their angular velocities in Section 2.2.2. Section 2.2.3 describes the dynamics of the attitude system using Euler's equation of motion.

\subsubsection{Inertia Matrices of the Satellite and the Fluid Rings}

It is considered a body frame $(\mathcal{B})$ attached to an artificial satellite, and the orientation of the axes $(X, Y, Z)$ in the body is assumed to coincide with its principal axes of inertia. The origin of the body-fixed frame $\mathcal{B}$ coincides with the center of mass of the satellite, and it is defined through the unit vectors $\hat{\boldsymbol{b}}_{1}, \hat{\boldsymbol{b}}_{2}$, and $\hat{\boldsymbol{b}}_{3}$. The inertia matrix of the satellite with respect to the body-fixed frame is:

$$
{ }^{\mathcal{B}}\left[\mathbf{I}_{s}\right]=\left[\begin{array}{ccc}
I_{s 1} & 0 & 0 \\
0 & I_{s 2} & 0 \\
0 & 0 & I_{s 3}
\end{array}\right]
$$

where $I_{s 1}, I_{s 2}$, and $I_{s 3}\left(\mathrm{~kg} \cdot \mathrm{m}^{2}\right)$ are the principal moments of inertia. The matrix ${ }^{\mathcal{B}}\left[\mathbf{I}_{s}\right]$, aside from containing the satellite inertia terms, also contains the inertia components of the fluid rings because the fluid rings center of mass does not coincide with the satellite center of mass.

For the fluid rings the obtaining of the inertia matrices is developed in Appendix A, thus the matrices of inertia for each fluid ring are written as:

$$
\begin{aligned}
\mathcal{B}_{\left[\mathbf{I}_{f 1}\right]} & =\left[\begin{array}{ccc}
I_{f 1 s} & 0 & 0 \\
0 & I_{f 1 t} & 0 \\
0 & 0 & I_{f 1 t}
\end{array}\right] \\
\mathcal{B}_{\left[\mathbf{I}_{f 2}\right]} & =\left[\begin{array}{ccc}
I_{f 2 t} & 0 & 0 \\
0 & I_{f 2 s} & 0 \\
0 & 0 & I_{f 2 t}
\end{array}\right] \\
\mathcal{B}_{\left[\mathbf{I}_{f 3}\right]} & =\left[\begin{array}{ccc}
I_{f 3 t} & 0 & 0 \\
0 & I_{f 3 t} & 0 \\
0 & 0 & I_{f 3 s}
\end{array}\right]
\end{aligned}
$$

where, for the $i$-th fluid ring, $I_{f i s}$ is the moment of inertia about its symmetric axis and $I_{\text {fit }}$ corresponds to the transverse axes.

\subsubsection{Angular Velocity}

The angular velocity of the frame $\mathcal{B}$ with respect to the inertial frame $\mathcal{N}$ (Figure 1) is represented by:

$$
{ }^{\mathcal{B}} \dot{\boldsymbol{q}}_{s}=\dot{q}_{s 1} \hat{\boldsymbol{b}}_{1}+\dot{q}_{s 2} \hat{\boldsymbol{b}}_{2}+\dot{q}_{s 3} \hat{\boldsymbol{b}}_{3}
$$

The relative angular velocities of the fluids inside the rings, with respect to the $\mathcal{B}$ frame, are written as:

$$
\begin{aligned}
{ }^{\mathcal{B}} \dot{\boldsymbol{q}}_{f 1} & =\left[\begin{array}{lll}
\dot{q}_{f 1} & 0 & 0
\end{array}\right]^{\top} \\
{ }^{\mathcal{B}} \dot{\boldsymbol{q}}_{f 2} & =\left[\begin{array}{lll}
0 & \dot{q}_{f 2} & 0
\end{array}\right]^{\top} \\
{ }^{\mathcal{B}} \dot{\boldsymbol{q}}_{f 3} & =\left[\begin{array}{lll}
0 & 0 & \dot{q}_{f 3}
\end{array}\right]^{\top}
\end{aligned}
$$


From (7), in the inertial frame $\mathcal{N}$, fluids' angular velocities are:

$$
\begin{aligned}
& \boldsymbol{\omega}_{f 1}=\dot{q}_{f 1} \hat{\boldsymbol{b}}_{1}+\dot{\boldsymbol{q}}_{s} \\
& \boldsymbol{\omega}_{f 2}=\dot{q}_{f 2} \hat{\boldsymbol{b}}_{2}+\dot{\boldsymbol{q}}_{s} \\
& \boldsymbol{\omega}_{f 3}=\dot{q}_{f 3} \hat{\boldsymbol{b}}_{3}+\dot{\boldsymbol{q}}_{s}
\end{aligned}
$$

where the shorthand notation ${ }^{\mathcal{B}} \dot{\boldsymbol{q}}_{s}=\dot{\boldsymbol{q}}_{s}$, from here on, will be used for simplicity.

Since the inertia matrices (5), and angular velocities (8) are identified, now they can be used in the Euler's equations of the following subsection in order to obtain the dynamics of the satellite.

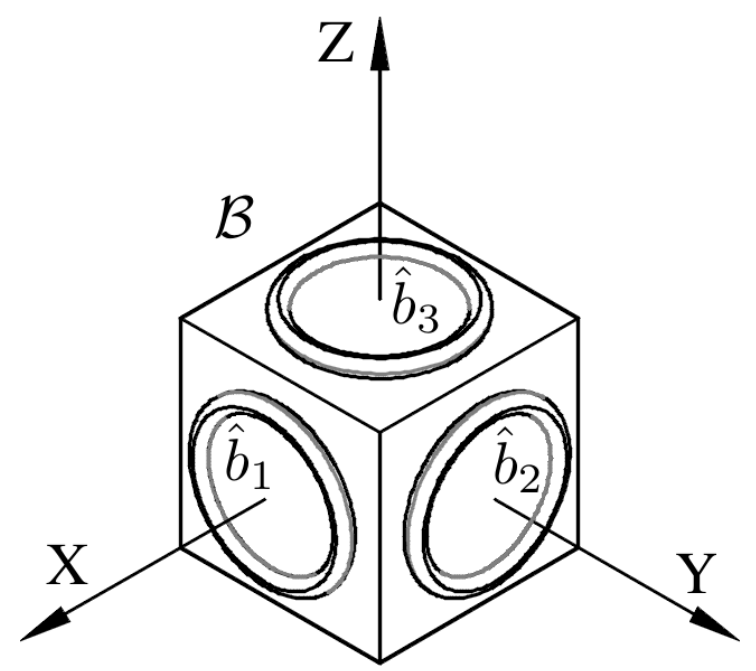

Figure 1. Satellite equipped with 3 fluid rings. The body frame attached to the satellite is called $\mathcal{B}$ and the unit vectors are $\hat{b}_{1}, \hat{b}_{2}$, and $\hat{b}_{3}$.

\subsubsection{Euler's Rotational Equations of Motion}

Using the transport theorem, Euler's equation is expressed as [33]:

$$
\frac{\mathcal{N}}{d t}=\frac{\mathcal{B}}{d t}+\dot{\boldsymbol{q}}_{s} \times \boldsymbol{H}=\boldsymbol{\tau}
$$

where, for a rotating body, $\mathcal{N}$ is an inertial frame, $\mathcal{B}$ is a frame fixed to the body, $[\mathbf{I}]$ is the inertial matrix of the body in terms of the $\mathcal{B}$ frame, $\dot{\boldsymbol{q}}_{s}$ is the angular velocity of the $\mathcal{B}$ frame with respect to the $\mathcal{N}$ frame in terms of the $\mathcal{B}$ frame, and $\tau(\mathrm{N} \cdot \mathrm{m})$ is the vector of external torques experienced by the body. Therefore, Equation (9) requires the angular momentum $\left(\boldsymbol{H}=[\mathbf{I}] \omega, \mathrm{kg} \cdot \mathrm{m}^{2} / \mathrm{s}\right)$ and its time derivative $\left(\frac{{ }^{\mathcal{B}} d \boldsymbol{H}}{d t}\right)$, both of them described below.

From Equations (4)-(6) and (8), the total angular momentum of the satellite and the fluid rings about the satellite's center of mass is:

$$
\begin{aligned}
\boldsymbol{H}= & I_{s 1} \dot{q}_{s 1} \hat{\boldsymbol{b}}_{1}+I_{s 2} \dot{q}_{s 2} \hat{\boldsymbol{b}}_{2}+I_{s 3} \dot{q}_{s 3} \hat{\boldsymbol{b}}_{3} \\
& +I_{f 1 s}\left(\dot{q}_{f 1}+\dot{q}_{s 1}\right) \hat{\boldsymbol{b}}_{1}+I_{f 1 t}\left(\dot{q}_{s 2}\right) \hat{\boldsymbol{b}}_{2}+I_{f 1 t}\left(\dot{q}_{s 3}\right) \hat{\boldsymbol{b}}_{3} \\
& +I_{f 2 t}\left(\dot{q}_{s 1}\right) \hat{\boldsymbol{b}}_{1}+I_{f 2 s}\left(\dot{q}_{f 2}+\dot{q}_{s 2}\right) \hat{\boldsymbol{b}}_{2}+I_{f 2 t}\left(\dot{q}_{s 3}\right) \hat{\boldsymbol{b}}_{3} \\
& +I_{f 3 t}\left(\dot{q}_{s 1}\right) \hat{\boldsymbol{b}}_{1}+I_{f 3 t}\left(\dot{q}_{s 2}\right) \hat{\boldsymbol{b}}_{2}+I_{f 3 s}\left(\dot{q}_{f 3}+\dot{q}_{s 3}\right) \hat{\boldsymbol{b}}_{3}
\end{aligned}
$$

To simplify Equation (10), the following matrix is introduced

$$
{ }^{\mathcal{B}}[\mathbf{I}]={ }^{\mathcal{B}}\left[\mathbf{I}_{s}\right]+{ }^{\mathcal{B}}\left[\mathbf{I}_{f 1}\right]+{ }^{\mathcal{B}}\left[\mathbf{I}_{f 2}\right]+{ }^{\mathcal{B}}\left[\mathbf{I}_{f 3}\right]
$$


Clearly, the matrix (11) is diagonal, and its elements are:

$$
\begin{aligned}
& I_{1}=I_{s 1}+I_{f 1 s}+I_{f 2 t}+I_{f 3 t} \\
& I_{2}=I_{s 2}+I_{f 1 t}+I_{f 2 s}+I_{f 3 t} \\
& I_{3}=I_{s 3}+I_{f 1 t}+I_{f 2 t}+I_{f 3 s}
\end{aligned}
$$

Using the moments of inertia from Equations (12) in (10), the total angular momentum is written in a more compact form as:

$$
\boldsymbol{H}=\left(I_{1} \dot{q}_{s 1}+h_{1}\right) \hat{\boldsymbol{b}}_{1}+\left(I_{2} \dot{q}_{s 2}+h_{2}\right) \hat{\boldsymbol{b}}_{2}+\left(I_{3} \dot{q}_{s 3}+h_{3}\right) \hat{\boldsymbol{b}}_{3}
$$

where the relative angular momenta of the fluid rings, about their center of mass, are defined as [35]:

$$
\begin{aligned}
& h_{1}=I_{f 1 s} \dot{q}_{f 1} \\
& h_{2}=I_{f 2 s} \dot{q}_{f 2} \\
& h_{3}=I_{f 3 s} \dot{q}_{f 3}
\end{aligned}
$$

From Euler's Equation (9), the time derivative of $\boldsymbol{H}$ is:

$$
\begin{aligned}
\mathcal{N}^{\mathcal{N}} \frac{d \boldsymbol{H}}{d t}= & \left(I_{1} \ddot{q}_{s 1}+\dot{h}_{1}\right) \hat{\boldsymbol{b}}_{1}+\left(I_{2} \ddot{q}_{s 2}+\dot{h}_{2}\right) \hat{\boldsymbol{b}}_{2}+\left(I_{3} \dot{q}_{s 3}+\ddot{h}_{3}\right) \hat{\boldsymbol{b}}_{3} \\
& +\dot{\boldsymbol{q}}_{s} \times\left[\left(I_{1} \dot{q}_{s 1}+h_{1}\right) \hat{\boldsymbol{b}}_{1}+\left(I_{2} \dot{q}_{s 2}+h_{2}\right) \hat{\boldsymbol{b}}_{2}+\left(I_{3} \dot{q}_{s 3}+h_{3}\right) \hat{\boldsymbol{b}}_{3}\right] \\
= & \boldsymbol{\tau}
\end{aligned}
$$

In Equation (15) do not appear explicitly the torques produced by the fluid rings. Then, the dynamics of each fluid ring can be isolated to represent their produced torques as follows [35]:

$$
\begin{aligned}
& u_{1}=I_{f 1 s} \ddot{q}_{s 1}+\dot{h}_{1} \\
& u_{2}=I_{f 2 s} \ddot{q}_{s 2}+\dot{h}_{2} \\
& u_{3}=I_{f 3 s} \ddot{q}_{s 3}+\dot{h}_{3}
\end{aligned}
$$

where the corresponding torque of the $i$-th fluid ring is $u_{i}(\mathrm{~N} \cdot \mathrm{m})$. To express Equation (15) in terms of the $u_{i}(16)$, new moments of inertia are defined as:

$$
\begin{aligned}
& I_{m 1}=I_{1}-I_{f 1 s} \\
& I_{m 2}=I_{2}-I_{f 2 s} \\
& I_{m 3}=I_{3}-I_{f 3 s}
\end{aligned}
$$

Substituting (17) and then (16) in (15), results:

$$
\begin{aligned}
\boldsymbol{\tau}= & I_{m 1} \ddot{q}_{s 1} \hat{\boldsymbol{b}}_{1}+I_{m 2} \ddot{q}_{s 2} \hat{\boldsymbol{b}}_{2}+I_{m 3} \ddot{q}_{s 3} \hat{\boldsymbol{b}}_{3} \\
& +\dot{\boldsymbol{q}}_{s} \times\left[\left(I_{1} \dot{q}_{s 1}+h_{1}\right) \hat{\boldsymbol{b}}_{1}+\left(I_{2} \dot{q}_{s 2}+h_{2}\right) \hat{\boldsymbol{b}}_{2}\right. \\
& \left.+\left(I_{3} \dot{q}_{s 3}+h_{3}\right) \hat{\boldsymbol{b}}_{3}\right]+u_{1} \hat{\boldsymbol{b}}_{1}+u_{2} \hat{\boldsymbol{b}}_{2}+u_{3} \hat{\boldsymbol{b}}_{3}
\end{aligned}
$$

The equation of motion (18) includes, explicitly, the torques produced by pumps of the fluid rings; therefore, the control laws can directly find the required fluid rings torques.

The vector of external torques $\tau$ in (18) is considered to be defined by the external gravity gradient torque. The gravity gradient torque vector acting on a rigid body, measured about its center of mass, is written as [33]: 


$$
\tau=\frac{3 G M_{e}}{2 R_{c}^{3}}\left[\begin{array}{c}
\left(I_{3}-I_{2}\right) \cos ^{2} \theta \sin 2 \phi \\
\left(I_{3}-I_{1}\right) \cos \phi \sin 2 \theta \\
\left(I_{1}-I_{2}\right) \sin \phi \sin 2 \theta
\end{array}\right]=\left[\begin{array}{l}
\tau_{1} \\
\tau_{2} \\
\tau_{3}
\end{array}\right]
$$

\subsection{Port-Hamiltonian Representation}

The dynamics of the attitude system shown in Section 2.2 along with the Hamiltonian function will allow for writing a model in the port-Hamiltonian form. A general description of port-Hamiltnonian systems is presented in Section 2.3.1 and the modeling of the fluid ring attitude system in a port-Hamiltonian form is written in Section 2.3.2.

\subsubsection{Port-Hamiltonian Systems}

The port-Hamiltonian systems have a geometric structure that derives from the interconnection of its subsystems and offers a framework for analysis, control, and simulation of complex physical systems. A central notion in the port-Hamiltonian systems is the Dirac structure whose basic property is power conservation. The notion of Dirac structure enables one to define Hamiltonian systems with algebraic constraints, so that any powerconserving interconnection of port-Hamiltonian systems defines again a port-Hamiltonian system [36-38].

A generalization of Hamiltonian systems with collocated inputs and outputs is [37]:

$$
\Sigma:=\left\{\begin{array}{l}
\dot{x}=[\mathbf{J}(x)] \frac{\partial \mathcal{H}}{\partial x}+[\mathbf{g}(x)] u \\
y=[\mathbf{g}(x)]^{\top} \frac{\partial \mathcal{H}}{\partial x}
\end{array}\right.
$$

where $x$ is the vector of energy variables, $\mathcal{H}$ is the Hamiltonian, $\boldsymbol{u}$, and $\boldsymbol{y}$ are the input and output corresponding to the control port, and the matrix $[\mathbf{J}(\boldsymbol{x})]$ is assumed to be skew-symmetric, this is:

$$
[\mathbf{J}(\boldsymbol{x})]=-[\mathbf{J}(\boldsymbol{x})]^{\top}
$$

2.3.2. Port-Hamiltonian Model for the Satellite-Fluid Rings System

The Hamiltonian is defined as [39]:

$$
\mathcal{H}(\boldsymbol{q}, \dot{\boldsymbol{q}}, t)=\sum_{i=1}^{n} \frac{\partial \mathcal{L}}{\partial \dot{q}_{i}} \dot{q}_{i}-\mathcal{L}=\boldsymbol{p}^{\top} \dot{\boldsymbol{q}}-\mathcal{L}
$$

where $q$ is the vector of generalized coordinates, $\mathcal{L}$ is the Lagrangian, and the vector of generalized torques $p$ is defined as:

$$
p=\frac{\partial \mathcal{L}}{\partial \dot{q}}
$$

The Lagrangian is defined as [39]:

$$
\mathcal{L}(\boldsymbol{q}, \dot{\boldsymbol{q}}, t)=T-U
$$

where $T$ and $U$ are the kinetic and potential energy of the system, respectively.

For the satellite, the angular kinetic energy is:

$$
T_{s}=\frac{1}{2} \dot{\boldsymbol{q}}_{s}^{\top}\left[\mathbf{I}_{s}\right] \dot{\boldsymbol{q}}_{s}
$$


and for the rings is written as:

$$
\begin{aligned}
T_{f 1} & =\frac{1}{2} \boldsymbol{\omega}_{f 1}^{\top}\left[\mathbf{I}_{f 1}\right] \boldsymbol{\omega}_{f 1} \\
T_{f 2} & =\frac{1}{2} \boldsymbol{\omega}_{f 2}^{\top}\left[\mathbf{I}_{f 2}\right] \boldsymbol{\omega}_{f 2} \\
T_{f 3} & =\frac{1}{2} \boldsymbol{\omega}_{f 3}^{\top}\left[\mathbf{I}_{f 3}\right] \boldsymbol{\omega}_{f 3}
\end{aligned}
$$

The sum of the Equations (25) and (26) is the kinetic energy of the satellite-fluid rings system, represented as:

$$
\begin{aligned}
T & =\frac{1}{2} \dot{\boldsymbol{q}}^{\top}\left[\begin{array}{cccccc}
I_{f 1 s} & 0 & 0 & I_{f 1 s} & 0 & 0 \\
0 & I_{f 2 s} & 0 & 0 & I_{f 2 s} & 0 \\
0 & 0 & I_{f 3 s} & 0 & 0 & I_{f 3 s} \\
I_{f s 1} & 0 & 0 & I_{1} & 0 & 0 \\
0 & I_{f s 2} & 0 & 0 & I_{2} & 0 \\
0 & 0 & I_{f s 3} & 0 & 0 & I_{3}
\end{array}\right] \dot{\boldsymbol{q}} \\
& =\frac{1}{2} \dot{\boldsymbol{q}}^{\top}[\mathbf{M}] \dot{\boldsymbol{q}}
\end{aligned}
$$

where $[\mathbf{M}]$ denotes the above matrix, and the vector $\dot{q} \in \mathbb{R}^{6}$ is:

$$
\begin{aligned}
& \dot{\boldsymbol{q}}=\left[\begin{array}{llllll}
\dot{q}_{f 1} & \dot{q}_{f 2} & \dot{q}_{f 3} & \dot{q}_{s 1} & \dot{q}_{s 2} & \dot{q}_{s 3}
\end{array}\right]^{\top} \\
& =\left[\begin{array}{ll}
\dot{\boldsymbol{q}}_{f}^{\top} & \dot{\boldsymbol{q}}_{s}^{\top}
\end{array}\right]^{\top}
\end{aligned}
$$

As it was stated earlier, the attitude of the satellite is described by the Euler angles $(\dot{\psi}, \dot{\theta}, \dot{\phi})$. Thus, a new vector of generalized coordinates is defined, and its time derivative is:

$$
\dot{\boldsymbol{q}}_{s}^{*}=\left[\begin{array}{lll}
\dot{\psi} & \dot{\theta} & \dot{\phi}
\end{array}\right]^{\top}
$$

Equation (1) relates the vectors $\dot{\boldsymbol{q}}_{s}$ and $\dot{\boldsymbol{q}}_{s}^{*}$. Therefore:

$$
\dot{\boldsymbol{q}}_{s}^{*}=\left[\mathbf{B}\left(\boldsymbol{q}_{s}^{*}\right)\right] \dot{\boldsymbol{q}}_{s}+\Omega \boldsymbol{a}\left(\boldsymbol{q}_{s}^{*}\right)
$$

where

$$
\boldsymbol{a}\left(\boldsymbol{q}_{s}^{*}\right)=\left[\begin{array}{c}
-\sin \theta \sin \psi / \cos \theta \\
-\cos \theta \cos \psi / \cos \theta \\
-\sin \psi / \cos \theta
\end{array}\right]
$$

Let $U$ be a potential function dependent on the Euler-angles $\left(\boldsymbol{q}_{s}^{*}\right)$, thus from (27) and (28), the Lagrangian (24) is written as:

$$
\begin{aligned}
& \mathcal{L}\left(\boldsymbol{q}_{s}^{*}, \dot{\boldsymbol{q}}_{f}, \dot{\boldsymbol{q}}_{s}\right)=\frac{1}{2}\left[\begin{array}{ll}
\dot{\boldsymbol{q}}_{f}^{\top} & \dot{\boldsymbol{q}}_{s}^{\top}
\end{array}\right][\mathbf{M}]\left[\begin{array}{c}
\dot{\boldsymbol{q}}_{f} \\
\dot{\boldsymbol{q}}_{s}
\end{array}\right]-U\left(\boldsymbol{q}_{s}^{*}\right) \\
& =\frac{1}{2} \dot{\boldsymbol{q}}^{\top}[\mathbf{M}] \dot{\boldsymbol{q}}-U\left(\boldsymbol{q}_{s}^{*}\right)
\end{aligned}
$$

Substituting (32) into (22), the Hamiltonian is written as:

$$
\mathcal{H}\left(\boldsymbol{q}_{s}^{*}, \boldsymbol{p}\right)=\frac{1}{2} \boldsymbol{p}^{\top}[\mathbf{M}]^{-1} \boldsymbol{p}+U\left(\boldsymbol{q}_{s}^{*}\right)
$$


where, from Equation (23) and (32), the generalized momentum vector is:

$$
\begin{aligned}
\boldsymbol{p}=\frac{\partial \mathcal{L}}{\partial \dot{\boldsymbol{q}}} & =[\mathbf{M}] \dot{\boldsymbol{q}} \\
& =\left[\begin{array}{ll}
\boldsymbol{p}_{f}^{\top} & \boldsymbol{p}_{s}^{\top}
\end{array}\right]^{\top}
\end{aligned}
$$

The elements of the vectors $\boldsymbol{p}_{f} \in \mathbb{R}^{3}$ are:

$$
\begin{aligned}
& p_{f 1}=I_{f 1 s} \dot{q}_{s 1}+h_{1} \\
& p_{f 2}=I_{f 2 s} \dot{q}_{s 2}+h_{2} \\
& p_{f 3}=I_{f 3 s} \dot{q}_{s 3}+h_{3}
\end{aligned}
$$

and the elements of the vectors $p_{s} \in \mathbb{R}^{3}$ are:

$$
\begin{aligned}
& p_{s 1}=I_{1} \dot{q}_{s 1}+h_{1} \\
& p_{s 2}=I_{2} \dot{q}_{s 2}+h_{2} \\
& p_{s 3}=I_{3} \dot{q}_{s 3}+h_{3}
\end{aligned}
$$

If the elements of the vector $\boldsymbol{p}_{f}$ are substituted into the equation representing the torques exerted by the fluid rings (16), it can be written:

$$
\begin{aligned}
& \dot{p}_{1}=u_{1} \\
& \dot{p}_{2}=u_{2} \\
& \dot{p}_{3}=u_{3}
\end{aligned}
$$

If the elements of the vector $\boldsymbol{p}_{s}$ are substituted into Euler's Equation (15), it results in:

$$
\dot{\boldsymbol{p}}_{s}=\boldsymbol{\tau}+[\tilde{\boldsymbol{p}}]_{s} \dot{\boldsymbol{q}}_{S}
$$

where the skew-symmetric tilde matrix for the $p_{s}$ vector is introduced as follows:

$$
[\tilde{\boldsymbol{p}}]_{s}=\left[\begin{array}{ccc}
0 & -p_{s 3} & p_{s 2} \\
p_{s 3} & 0 & -p_{s 1} \\
-p_{s 2} & p_{s 1} & 0
\end{array}\right]
$$

Now, for the partial derivatives of the Hamiltonian (33).

$$
\frac{\partial \mathcal{H}\left(\boldsymbol{q}_{s}^{*}, \boldsymbol{p}\right)}{\partial \boldsymbol{p}}=[\mathbf{M}]^{-1} \boldsymbol{p}
$$

from (34) we know that also:

$$
\dot{q}=\left[\mathbf{M}_{s}\right]^{-1} p
$$

thus:

$$
\dot{\boldsymbol{q}}=\frac{\partial \mathcal{H}\left(\boldsymbol{q}_{s}^{*}, \boldsymbol{p}\right)}{\partial p}
$$

or

$$
\left[\begin{array}{c}
\dot{\boldsymbol{q}}_{f} \\
\dot{\boldsymbol{q}}_{s}
\end{array}\right]=\left[\begin{array}{l}
\frac{\partial \mathcal{H}}{\partial p_{f}} \\
\frac{\partial \mathcal{H}}{\partial p_{s}}
\end{array}\right]
$$


If instead of $\boldsymbol{q}_{s}$, the vector $\boldsymbol{q}_{s}^{*}$ (Euler angles) is used, from (30) and (43) we can write:

$$
\left[\begin{array}{c}
\dot{\boldsymbol{q}}_{f} \\
\dot{\boldsymbol{q}}_{s}^{*}
\end{array}\right]=\left[\begin{array}{c}
\frac{\partial \mathcal{H}}{\partial p_{f}} \\
{\left[\mathbf{B}\left(\boldsymbol{q}_{s}^{*}\right)\right] \frac{\partial \mathcal{H}}{\partial p_{s}}}
\end{array}\right]+\left[\begin{array}{c}
0 \\
0 \\
0 \\
\boldsymbol{a}\left(\boldsymbol{q}_{s}^{*}\right)
\end{array}\right] \Omega
$$

The Hamiltonian (33) does not depend on $\boldsymbol{q}_{f}$, thus:

$$
\frac{\partial \mathcal{H}}{\partial q_{f}}=0
$$

As the external gravity gradient torque (19) comes from a conservative force, we related it with the gradient of the Hamiltonian $\frac{\partial \mathcal{H}}{\partial q_{s}^{*}}$ and, to keep the geometric structure of the port-Hamiltonian system, is written as:

$$
\boldsymbol{\tau}=-[\mathbf{B}(\psi, \theta, \phi)]^{\top} \frac{\partial \mathcal{H}}{\partial \boldsymbol{q}_{s}^{*}}
$$

where, solving for $\frac{\partial \mathcal{H}}{\partial \boldsymbol{q}_{s}^{*}}$, results:

$$
\frac{\partial \mathcal{H}}{\partial \boldsymbol{q}_{s}^{*}}=\left[\begin{array}{c}
0 \\
-\sin 2 \theta\left(\frac{I_{2}-2 I_{1}+I_{3}-I_{2} \cos 2 \phi+I_{3} \cos 2 \phi}{4}\right) \\
\cos \phi \cos ^{2} \theta \sin \phi\left(I_{2}-I_{3}\right)
\end{array}\right] \frac{3 G M_{e}}{R_{c}^{3}}
$$

From Equations (37), (38), (44) and (46), we can represent the model of the system in the port-Hamiltonian form:

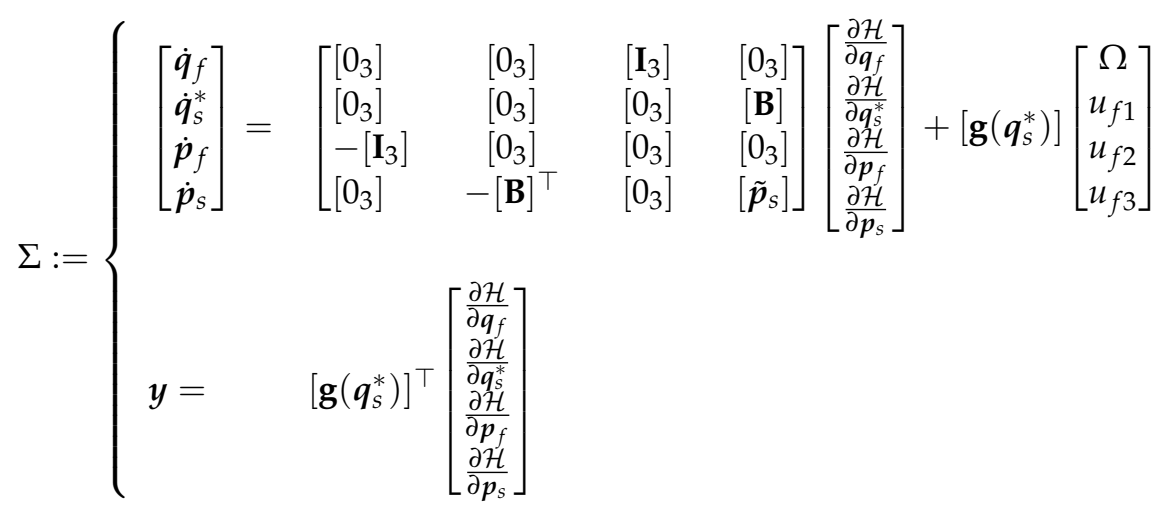

where

$$
\left[\mathbf{g}\left(\boldsymbol{q}_{s}^{*}\right)\right]=\left[\begin{array}{cc}
{\left[0_{3 \times 1}\right]} & {\left[0_{3}\right]} \\
{[\boldsymbol{a}]} & {\left[0_{3}\right]} \\
{\left[0_{3 \times 1}\right]} & {\left[\mathbf{I}_{3}\right]} \\
{\left[0_{3 \times 1}\right]} & {\left[0_{3}\right]}
\end{array}\right],
$$

$\left[0_{3}\right]$ is a $3 \times 3$ null matrix, and $\left[\mathbf{I}_{3}\right]$ is the $3 \times 3$ identity matrix. The model (48) comprises two ports, the energy-storage and control port. The port variables corresponding to the energy-storage port are:

$$
\begin{aligned}
& f_{s}=-\left[\begin{array}{llll}
\dot{\boldsymbol{q}}_{f} & \dot{\boldsymbol{q}}_{s}^{*} & \dot{\boldsymbol{p}}_{f} & \dot{\boldsymbol{p}}_{s}
\end{array}\right]^{\top} \\
& \boldsymbol{e}_{s}=\left[\begin{array}{llll}
\frac{\partial \mathcal{H}}{\partial \boldsymbol{q}_{f}} & \frac{\partial \mathcal{H}}{\partial \boldsymbol{q}_{s}^{*}} & \frac{\partial \mathcal{H}}{\partial p_{f}} & \frac{\partial \mathcal{H}}{\partial p_{s}}
\end{array}\right]^{\top}
\end{aligned}
$$


while the variables of the control port are:

$$
\begin{aligned}
\boldsymbol{e}_{c} & =\boldsymbol{y} \\
f_{c} & =\left[\begin{array}{llll}
\Omega & u_{1} & u_{2} & u_{3}
\end{array}\right]^{\top}
\end{aligned}
$$

The Dirac structure of the port-Hamiltonian systems satisfies the power balance [37]:

$$
\boldsymbol{e}_{s}^{\top} f_{s}+\boldsymbol{e}_{c}^{\top} f_{c}=0
$$

where $\boldsymbol{e}_{s}^{\top} f_{s}\left(\boldsymbol{e}_{c}^{\top} f_{c}\right)$ equals the power corresponding to the storage (control) port. Thus, from (48), (50), and (52) the energy balance is written as:

$$
\frac{d}{d t} \mathcal{H}=\frac{\partial^{\top} \mathcal{H}}{\partial x} \dot{x}=-e_{s}^{\top} f_{s}=e_{c}^{\top} f_{c}
$$

where

$$
\dot{x}=\left[\begin{array}{llll}
\dot{\boldsymbol{q}}_{f}^{\top} & \dot{\boldsymbol{q}}_{s}^{* \top} & \dot{\boldsymbol{p}}_{f}^{\top} & \dot{\boldsymbol{p}}_{s}^{\top}
\end{array}\right]
$$

The port-Hamiltonian system (48) is defined with respect to the modulated Dirac structure $\mathcal{D}$ written as:

$$
\mathcal{D}=\left\{\left(\boldsymbol{f}_{s}, \boldsymbol{e}_{s}, \boldsymbol{f}_{c}, \boldsymbol{e}_{c}\right) \mid \boldsymbol{e}_{c}=\left[\mathbf{g}^{\top}\left(\boldsymbol{q}_{s}^{*}\right)\right] \boldsymbol{e}_{s},-f_{s}=[\mathbf{J}(\boldsymbol{x})] \boldsymbol{e}_{s}+\left[\mathbf{g}\left(\boldsymbol{q}_{s}^{*}\right)\right] f_{c}\right\}
$$

\section{Results}

The following subsections describe the results concerning the attitude model in portHamiltonian representation and the model for the torques of the fluid rings. For the port Hamiltonian system presented in this work, a simulation comparing the outputs (Euler angles) is given with those of Euler's equations, aiming to show that the two models are equivalent representations of the same physical system. Regarding the fluid rings torques, an experimental prototype of a fluid ring is presented, that is used to collect data and then implement linear regression analysis to model the torque of the fluid rings. These torques are to be substituted in the port-Hamiltonian system, thus completing the attitude model.

\subsection{Comparing the Euler Equations and the Port-Hamiltonian System}

In order to validate the port-Hamiltonian system (48), a comparison with Euler's Equations (18) is made and shown as an open-loop simulation in Figures 2-4. The software used in the simulation is SIMULINK ${ }^{\circledR}$ of MATLAB $^{\circledR}$ (R2020b Update 4 (9.9.0.1570001)/The MathWorks, Inc., Natick, MA, USA) with the solver Runge-Kuta, and a fixed-step size of $1 \mathrm{~ms}$. The initial conditions for the Euler angles $(\psi, \theta, \phi)$ are arbitrarily chosen as $0.3 \mathrm{rad}, 0.2 \mathrm{rad}$, and $0.1 \mathrm{rad}$, respectively. The three fluid rings are assumed to be exerting a constant torque and it is considered the effect of the gravitational torque for a satellite in a geostationary orbit. The parameters shown in Table 1 are used in the simulations. 
Table 1. Simulation parameters.

\begin{tabular}{ccc}
\hline Parameter & Value & Units \\
\hline$I_{\text {fis }}$ for $i=1,2,3$ & 0.0061 & $\mathrm{~kg} \cdot \mathrm{m}^{2}$ \\
\hline$I_{\text {fit }}$, for $i=1,2,3$ & 0.0030 & $\mathrm{~kg} \cdot \mathrm{m}^{2}$ \\
\hline$I_{s 1}$ & 0.4254 & $\mathrm{~kg} \cdot \mathrm{m}^{2}$ \\
\hline$I_{s 2}$ & 0.5054 & $\mathrm{~kg} \cdot \mathrm{m}^{2}$ \\
\hline$I_{s 3}$ & 0.4254 & $\mathrm{~kg} \cdot \mathrm{m}^{2}$ \\
\hline$G$ & $6.6740 \times 10^{-11}$ & $\mathrm{~m}^{3} \cdot \mathrm{kg}-1 \cdot \mathrm{s}^{-2}$ \\
\hline$M_{e}$ & $5.9722 \times 10^{24}$ & $\mathrm{~kg}$ \\
\hline$R_{c}$ & $42,164,000$ & $\mathrm{~m}$ \\
\hline$u_{1}$ & 0.03 & $\mathrm{~N} \cdot \mathrm{m}$ \\
\hline$u_{2}$ & 0.02 & $\mathrm{~N} \cdot \mathrm{m}$ \\
\hline$u_{3}$ & 0.01 & $\mathrm{~N} \cdot \mathrm{m}$ \\
\hline
\end{tabular}

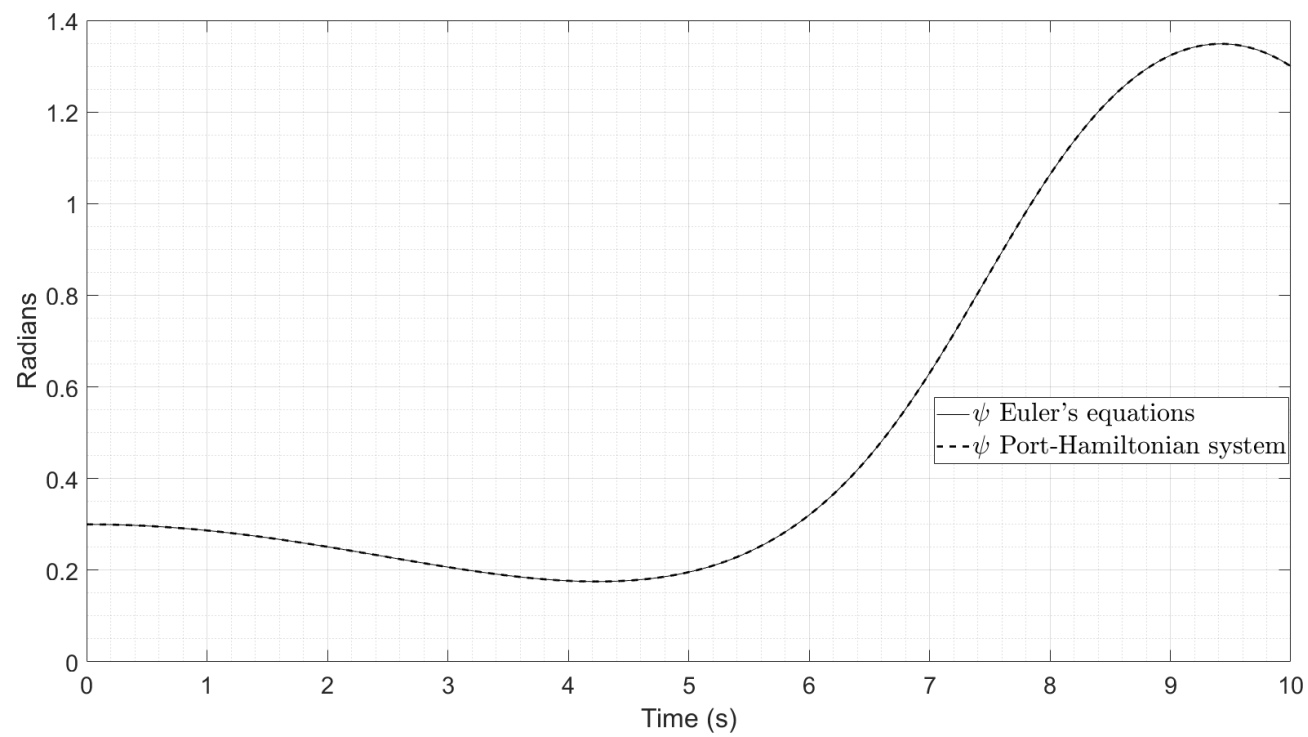

Figure 2. Comparison of the yaw angle $(\psi)$ between the Euler's equations and the portHamiltonian system. 


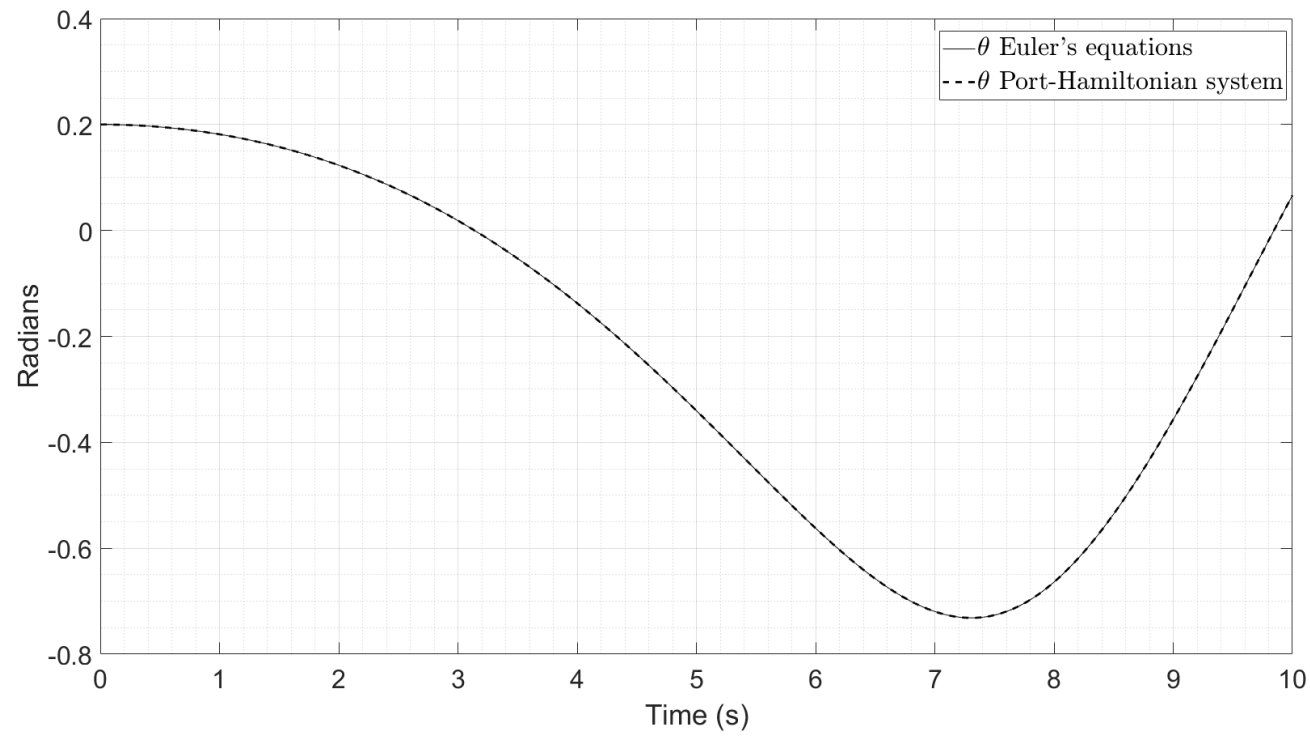

Figure 3. Comparison of the pitch angle $(\theta)$ between the Euler's equations and the portHamiltonian system.

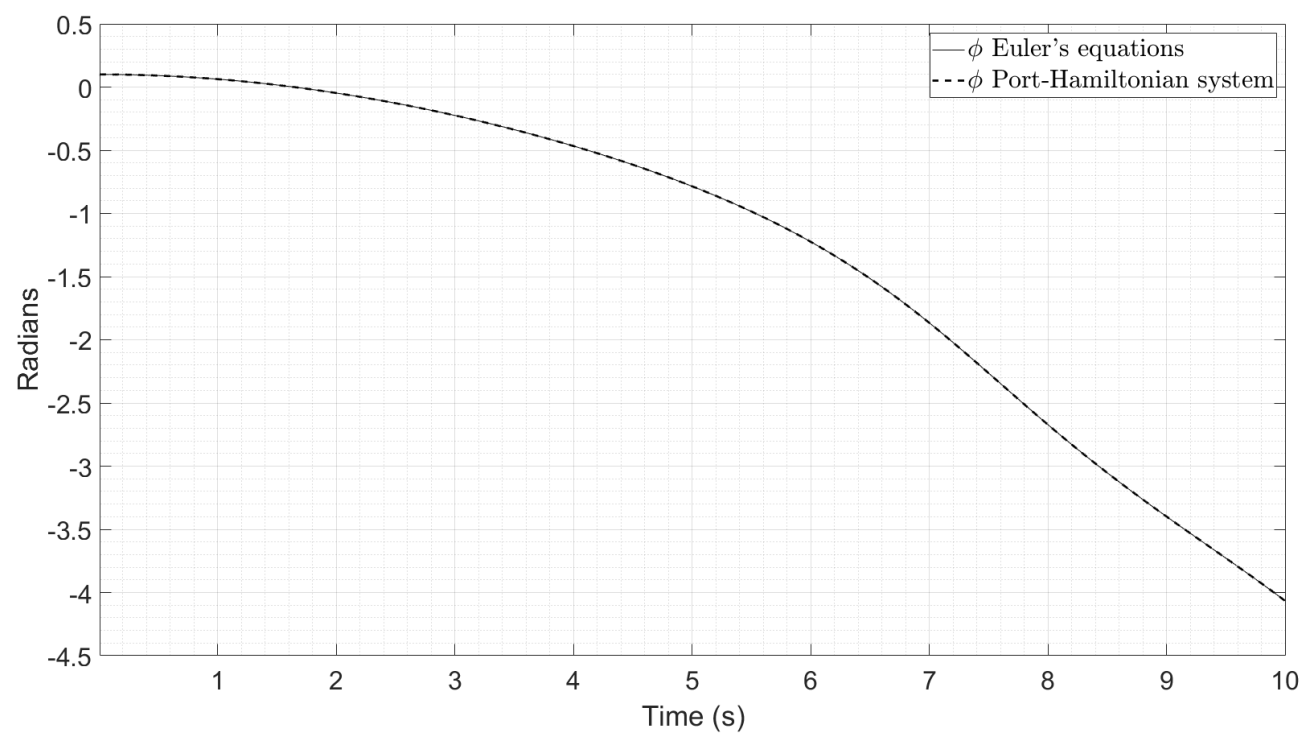

Figure 4. Comparison of the roll angle $(\phi)$ between the Euler's equations and the portHamiltonian system.

The Euler angles simulated using Euler's equations are represented by continuous lines in the latter figures, and the dashed lines correspond to the same angles but are obtained from the port-Hamiltonian system. As can be seen in the figures, both models concur.

\subsection{Fluid Rings' Torques from an Experimental Prototype}

The torque inputs $u_{f 1}, u_{f 2}$, and $u_{f 3}$ in Equation (48) depend on the flow in the fluid ring, which can be either laminar or turbulent. Fluid dynamics are complex, especially in turbulent flows. Therefore, as the flow near the fluid ring mechanical pump is presumably turbulent, it was considered a phenomenological approach to model the torque. Otherwise, it would have been needed to consider complex correlations involving fluid mechanics.

The prototype that is shown in Figure 5 was used to collect data on the volume flow rate as the supplied voltage to the pump is varied. This prototype comprises the following elements: 
- A water pump model JT-750D4 12V

- A water flow sensor model YF-201 with maximum capacity of measurement of 720 liter

- $\quad$ An Arduino ${ }^{\circledR}$ Uno microcontroller

- A laptop computer

- A 3/4" diameter water hose

- A laboratory DC power supply

- An ammeter

There were several measurements collected, increasing the voltage $0.5 \mathrm{~V}$ each time. The average value of the volume flow rate and the electric current is shown in Table 2.

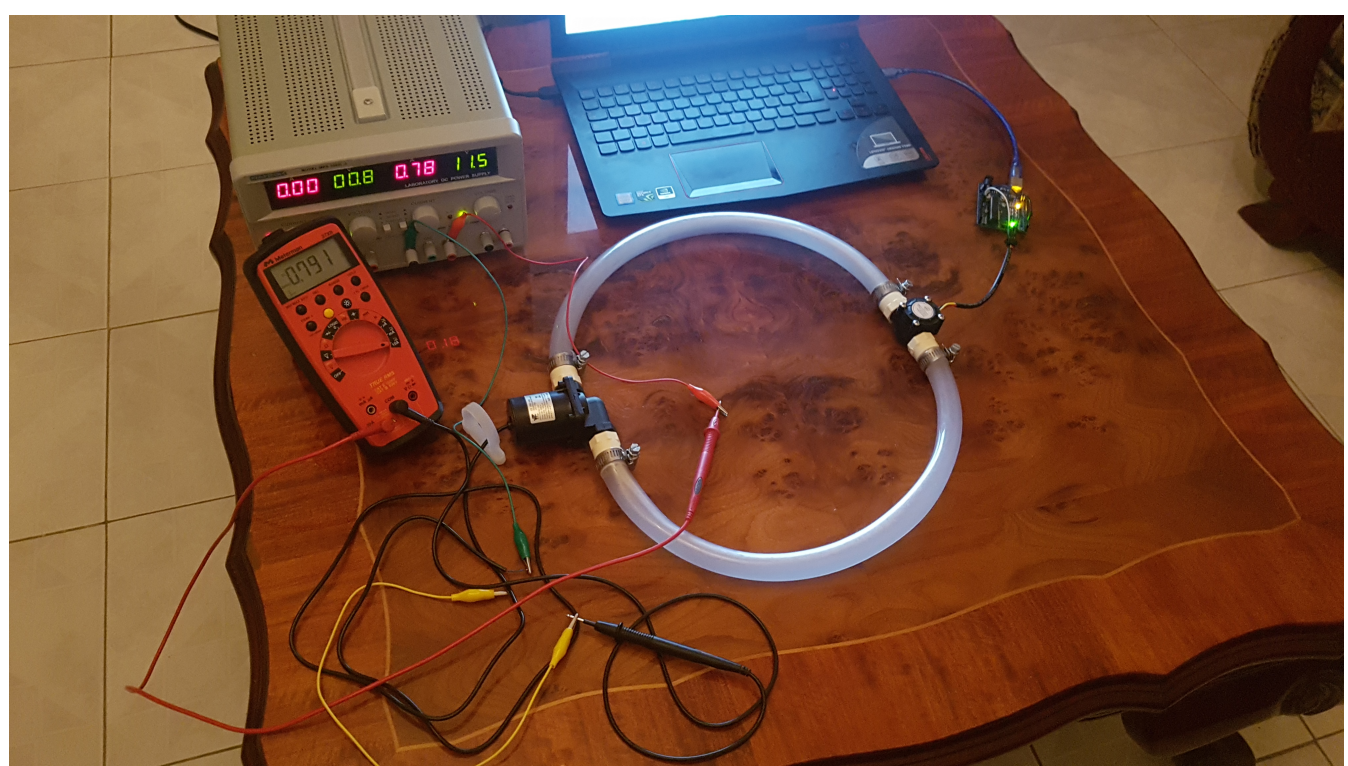

Figure 5. Experimental prototype.

Table 2. Measurements of the volume flow rate and electric current while varying the voltage.

\begin{tabular}{ccc}
\hline Voltage $(\mathbf{V})$ & Volume Flow Rate $\left(\mathrm{m}^{3} / \mathbf{s}\right)$ & Electric Current (A) \\
\hline 6.0 & 0.0000722 & 0.360 \\
\hline 6.5 & 0.0000772 & 0.400 \\
\hline 7.0 & 0.0000822 & 0.457 \\
\hline 7.5 & 0.0000872 & 0.503 \\
\hline 8.0 & 0.0000922 & 0.543 \\
\hline 8.5 & 0.0000964 & 0.580 \\
\hline 9.0 & 0.0001014 & 0.630 \\
\hline 9.5 & 0.0001047 & 0.667 \\
\hline 10.0 & 0.0001097 & 0.710 \\
\hline 10.5 & 0.0001147 & 0.750 \\
\hline 11.0 & 0.0001172 & 0.787 \\
\hline 11.5 & 0.0001214 & 0.823 \\
\hline 12.0 & 0.0001247 & 0.867 \\
\hline
\end{tabular}

Then, from the following equation for the torque:

$$
\tau_{p}=I_{p} \dot{\omega}_{p}
$$


where $\tau_{p}(\mathrm{~N} \cdot \mathrm{m})$ is the torque generated by the pumped fluid in the ring, $I_{p}\left(\mathrm{~kg} \cdot \mathrm{m}^{2}\right)$ is the moment of inertia of the fluid ring about its symmetry axis, and $\omega_{p}(\mathrm{rad} / \mathrm{s})$ is the angular velocity of the fluid in the ring.

Since the volume flow rate $Q\left(\mathrm{~m}^{3} / \mathrm{s}\right)$ is equal to the velocity of the fluid $v(\mathrm{~m} / \mathrm{s})$ times the cross-sectional area of the flow $A_{c}\left(\mathrm{~m}^{2}\right)$ [40], then:

$$
Q=v A_{c}
$$

where the velocity of the fluid $v$ is:

$$
v=\omega_{p} r
$$

Substituting Equation (58) in (57), and rearranging, results in:

$$
\omega_{p}=\frac{Q}{A_{c} r}
$$

As the cross-sectional area and the radius are constant, then:

$$
\dot{\omega}_{p}=\frac{1}{A_{c} r} \frac{d Q}{d t}
$$

Substituting Equation (60) in (56) allows us to write the torque $\left(\tau_{p}\right)$ as:

$$
\tau_{p}=\frac{I_{p}}{A_{c} r} \frac{d Q}{d t}
$$

From the data in Table 2, and using the method of least squares, the relation between the volume flow rate $Q$ and the electric current $i(\mathrm{~A})$ is represented by the following linear equation:

$$
Q=0.10437 \times 10^{-3} i+0.03457 \times 10^{-3}
$$

Figure 6 shows the plot of Equation (62).

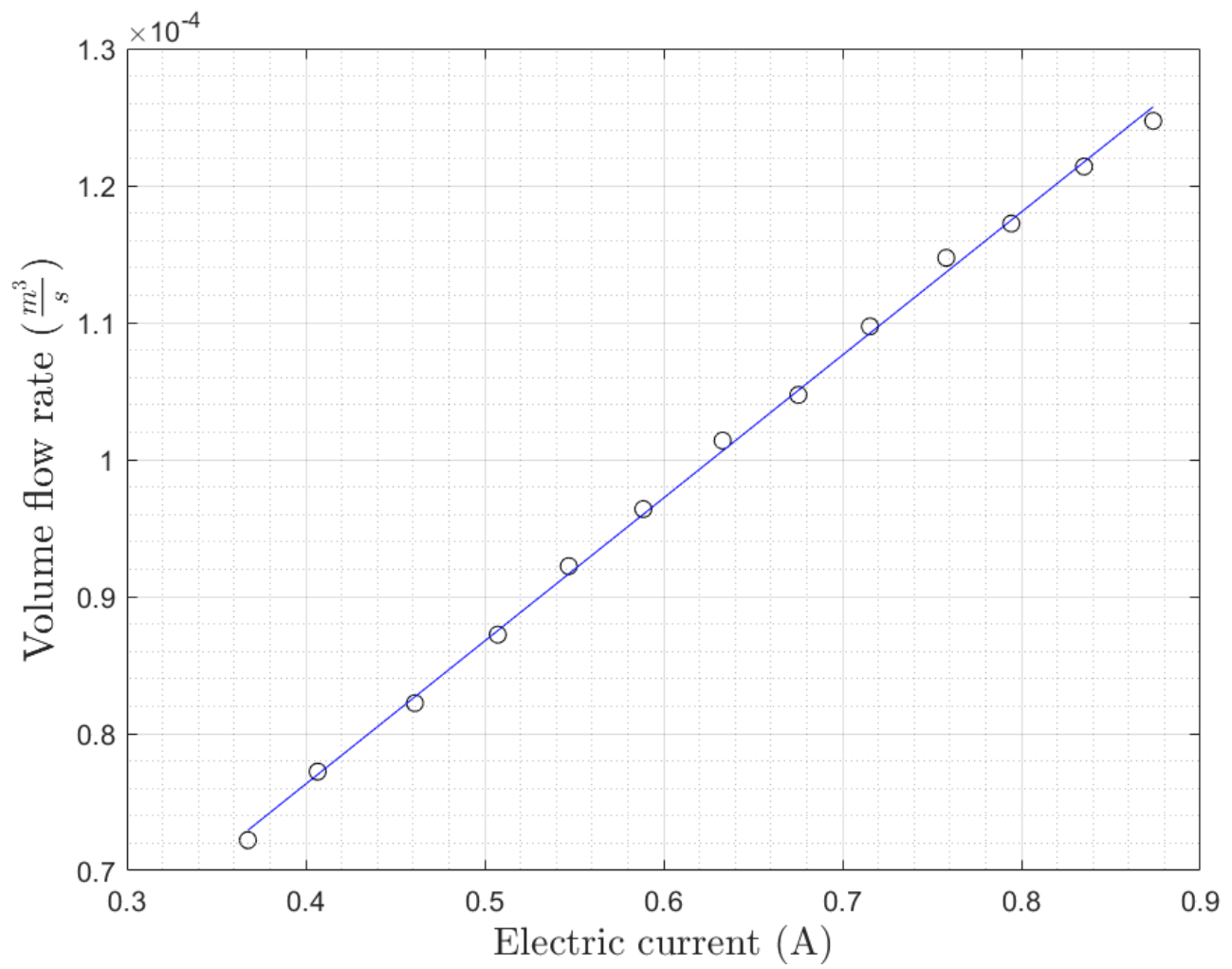

Figure 6. Relation between electric current and volume flow rate. 
The time derivative of $Q$ is defined by the chain rule as:

$$
\frac{d Q}{d t}=\frac{d Q}{d i} \frac{d i}{d t}
$$

From Equations (62) and (63), the Equation (61) is written as:

$$
\tau_{p}=\frac{I_{p}}{A_{c} r} 0.10437 \times 10^{-3} \frac{d i}{d t}
$$

Now the torque exerted by the fluid rings is described as a function of the varying electrical current.

The torque $u_{f i}$ in Equation (48) represents the torque produced by the fluid ring. The experimental torque of the fluid ring obtained with the prototype is $\tau_{p}$. Then if it is considered that all the fluid rings have the same dimensions as the one in the experiment (64), the torque produced by each fluid ring is represented as:

$$
u_{f i}=K_{f} \frac{d i_{i}}{d t}
$$

where $K_{f}=\frac{I_{p}}{A_{c} r} 0.10437 \times 10^{-3}$ and $i_{i}$ is the electrical current supplied by the pump of the $i$-th fluid ring.

Perhaps, due to limitations of the experimental equipment used and the concrete choice on the experimental electric current range, model (62) turned out to be a linear one. However, notice that even for a non-linear empirical relation $Q=Q(i)$, obtained from regression analysis, it is still possible, from (64), to find an empirical formula for the pump torque as:

$$
\tau=\frac{I_{p}}{A_{c} r} \frac{d Q}{d i} \frac{d i}{d t},
$$

without considering the complexities of the turbulent flow in the fluid ring system.

\section{Conclusions}

In this work, a dynamical model is developed for a fluid ring attitude system and the torques exerted by the fluid rings. Since the model for the attitude system is presented in a port-Hamiltonian form, it is possible to apply the powerful tools for analysis, control, and simulation, characteristically of such representation. Furthermore, because the powerconserving interconnection of port-Hamiltonian systems again defines a port-Hamiltonian system then, it is possible the developing a more complex system from the interconnection of simpler parts if the aim is to enrich the model in the future.

For the fluid rings, the dynamics are complex, hence the prediction of the flow and the exact calculation of energy losses are very difficult to achieve [41,42]. Therefore, in this work, we decided to use a simpler model that still yields satisfactory results. This model obtained by using linear regression analysis [43] is relatively simple and has reasonable accuracy. Nevertheless, the regression analysis could be applied to model different kinds of pumps and working fluids that yield nonlinear relations between the torque and the applied current. Since this model describes the overall torque exerted by each ring, it is unnecessary to consider head losses (as the frictional one), and consequently, there is no need to highlight if the flow is laminar or turbulent.

Author Contributions: Conceptualization, J.C.A.T. and H.V.-R.; Methodology, J.C.A.T. and C.E.C.; Resources, C.E.C.; Validation, J.C.A.T.; Writing-review \& editing, C.E.C. and H.V.-R. All authors have read and agreed to the published version of the manuscript.

Funding: This research received no external funding.

Institutional Review Board Statement: Not applicable.

Informed Consent Statement: Not applicable. 
Data Availability Statement: Not applicable.

Acknowledgments: Authors thanks to CONACYT (México) under scholarship number 779292 and PROINPEP 2021 under project no. 259988.

Conflicts of Interest: The authors declare no conflict of interest.

\section{Appendix A. Inertia Matrix of the Fluid Rings}

For the fluid rings, it is considered a frame attached to each one $\left(\mathcal{R}_{i}\right)$ (Figure A1). The 1 -st fluid ring has attached the $\left(\mathcal{R}_{1}\right)$ frame, and its origin coincides with the center of mass of the ring.

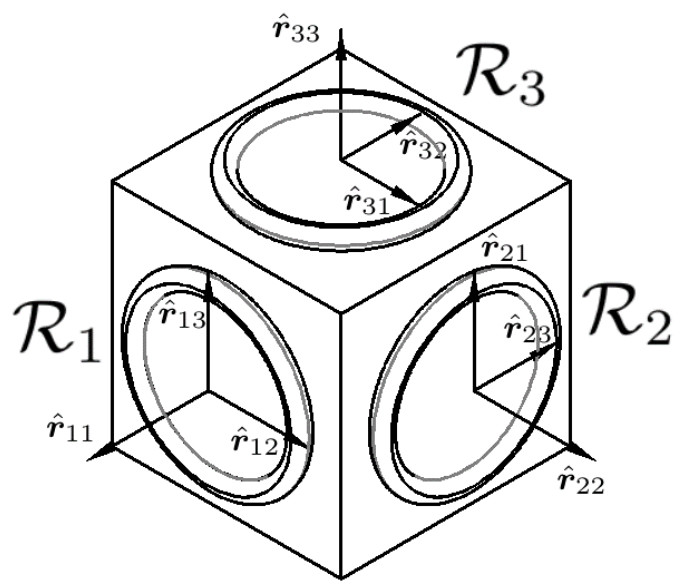

Figure A1. It is considered a frame attached to each fluid $\operatorname{ring} \mathcal{R}_{1}, \mathcal{R}_{2}$, and $\mathcal{R}_{3}$.

Fluid rings are modeled assuming they are thin. The $i$-th ring (Figure A2) is parametrized as:

$$
\boldsymbol{r}_{1}=0 \hat{r}_{11}+r_{1} \cos \phi_{1} \hat{r}_{12}+r_{1} \sin \phi_{1} \hat{r}_{13}
$$

Thus, the components of the vector $\boldsymbol{r}_{1}$ are:

$$
r_{11}=0, \quad r_{12}=r_{1} \cos \phi_{1}, \quad r_{13}=r_{1} \sin \phi_{1}
$$

The differential of the arc length $s_{1}$ is defined as:

$$
d s_{1}=r_{1} d \phi_{1}
$$

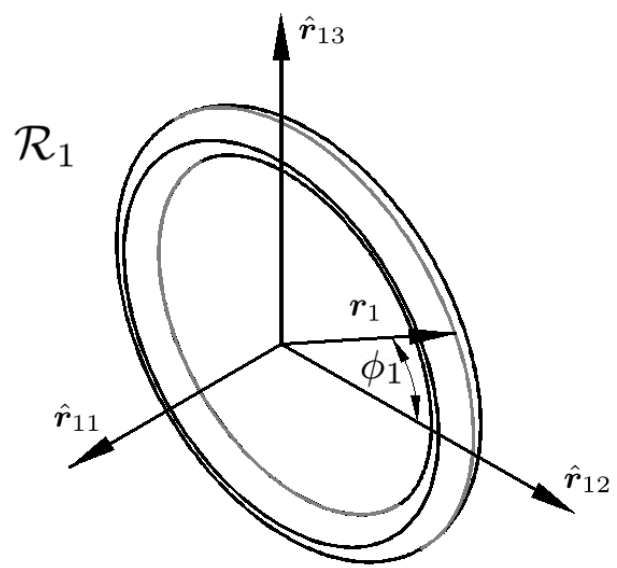

Figure A2. Unit vectors of the frame fixed to the fluid ring 1 . 
Then, the components of the inertia matrix for the 1-st fluid ring, with respect to the origin of the frame $\mathcal{R}_{1}$, are defined as [44,45]:

$$
{ }^{\mathcal{R}_{1}} I_{f 1_{i j}}=\int_{0}^{2 \pi}\left[r_{1}^{2} \delta_{i j}-r_{1 i} r_{1 j}\right] \lambda_{1} r_{1} d \phi_{1}
$$

where $\lambda_{1}$ is the linear density of the 1-st fluid ring, and the notation $r^{2 n}=\left(\boldsymbol{r}^{\top} \boldsymbol{r}\right)^{n}$ is given.

Substituting the components of the vector $r_{1}$ (A2) it is found:

$$
I_{f 1 x x}=m_{f 1} r_{1}^{2}, \quad I_{f 1 y y}=I_{f 1 z z}=\frac{1}{2} m_{f 1} r_{1}^{2}
$$

where $m_{f 1}=2 \pi \lambda_{1} r_{1}$ is the mass of the 1-st fluid ring. We can define its moments of inertia as:

$$
I_{f 1 s}=m_{f 1} r_{1}^{2}, \quad I_{f 1 t}=\frac{1}{2} m_{f 1} r_{1}^{2}
$$

where $I_{f 1 s}$ is the moment of inertia about the symmetric axis and $I_{f 1 t}$ is the one corresponding to the transverse axes. Then, the inertia matrices for the three fluid rings are:

$$
\begin{aligned}
\left.\mathcal{B}_{[} \mathbf{I}_{f 1}\right] & =\left[\begin{array}{ccc}
I_{f 1 s} & 0 & 0 \\
0 & I_{f 1 t} & 0 \\
0 & 0 & I_{f 1 t}
\end{array}\right] \\
\left.\mathcal{B}_{[} \mathbf{I}_{f 2}\right] & =\left[\begin{array}{ccc}
I_{f 2 t} & 0 & 0 \\
0 & I_{f 2 s} & 0 \\
0 & 0 & I_{f 2 t}
\end{array}\right] \\
\left.\mathcal{B}_{[} \mathbf{I}_{f 3}\right] & =\left[\begin{array}{ccc}
I_{f 3 t} & 0 & 0 \\
0 & I_{f 3 t} & 0 \\
0 & 0 & I_{f 3 s}
\end{array}\right]
\end{aligned}
$$

As the rings are axisymmetric about the $\hat{\boldsymbol{r}}_{i i}=\hat{\boldsymbol{b}}_{i}$ axes, then ${ }^{\mathcal{R}_{i}}\left[\mathbf{I}_{f i}\right]={ }^{\mathcal{B}}\left[\mathbf{I}_{f i}\right]$.

\section{References}

1. Froelich, R.; Papapoff, H. Reaction wheel attitude control for space vehicles. IRE Trans. Autom. Control 1959, 4, 139-149. [CrossRef]

2. Dertouzos, M.; Roberge, J. High-Capacity Reaction-Wheel Attitude Control. IEEE Trans. Appl. Ind. 1964, 83, 99-104. [CrossRef]

3. Macala, G.A. Design of the reaction wheel attitude control system for the Cassini spacecraft. In Proceedings of the 12th AAS/AIAA Space Flight Mechanics Meeting, San Antonio, TX, USA, 27-30 January 2002; p. 0122

4. Ismail, Z.; Varatharajoo, R. A study of reaction wheel configurations for a 3-axis satellite attitude control. Adv. Space Res. 2010, 45, 750-759. [CrossRef]

5. Marsh, H.C.; Karpenko, M.; Gong, Q. Relationships between maneuver time and energy for reaction wheel attitude control. J. Guid. Control Dyn. 2018, 41, 335-348. [CrossRef]

6. King, J.T. Increasing agility in orthogonal reaction wheel attitude control systems. Acta Astronaut. 2020, 177, 673-683. [CrossRef]

7. Singh, S.N.; Bossart, T.C. Exact feedback linearization and control of space station using CMG. IEEE Trans. Autom. Control 1993, 38, 184-187. [CrossRef]

8. Hoelscher, B. Optimal open-loop and feedback control using single gimbal control moment gyroscopes. J. Astronaut. Sci. 1994, 42, 189-206.

9. MacKunis, W.; Dupree, K.; Fitz-Coy, N.; Dixon, W. Adaptive satellite attitude control in the presence of inertia and CMG gimbal friction uncertainties. J. Astronaut. Sci. 2008, 56, 121-134. [CrossRef]

10. MacKunis, W.; Leve, F.; Patre, P.; Fitz-Coy, N.; Dixon, W. Adaptive neural network-based satellite attitude control in the presence of CMG uncertainty. Aerosp. Sci. Technol. 2016, 54, 218-228. [CrossRef]

11. Wiśniewski, R.; Blanke, M. Fully magnetic attitude control for spacecraft subject to gravity gradient. Automatica 1999, 35, 1201-1214. [CrossRef]

12. Santoni, F.; Zelli, M. Passive magnetic attitude stabilization of the UNISAT-4 microsatellite. Acta Astronaut. 2009, 65, 792-803. [CrossRef]

13. Desouky, M.A.; Abdelkhalik, O. Improved spacecraft magnetic attitude maneuvering. J. Spacecr. Rocket. 2019, 56, 1611-1623. [CrossRef]

14. Maynard, R.S. Fluidic Momentum Controller. US Patent 4,776,541, 11 October 1988. 
15. Varatharajoo, R.; Kahle, R.; Fasoulas, S. Approach for combining spacecraft attitude and thermal control systems. J. Spacecr. Rocket. 2003, 40, 657-664. [CrossRef]

16. Shan, X.; Chen, X.; Geng, Y.; Zhang, S. Small satellite attitude control based on mechanically-pumped fluid loops. In Proceedings of the 2011 6th IEEE Conference on Industrial Electronics and Applications, Beijing, China, 21-23 June 2011; pp. 149-153.

17. Kelly, A.; Mc Chesney, C.; Smith, P.; Waltena, S. A Performance Test of a Fluidic Momentum Controller in Three Axes. In NASA Report; The University of Texas at Austin: Austin, TX, USA, 2004.

18. Kumar, K. Satellite attitude stabilization using fluid rings. Acta Mech. 2009, 208, 117-131. [CrossRef]

19. Nobari, N.; Misra, A. Satellite attitude stabilization using four fluid rings in a pyramidal configuration. In Proceedings of the AIAA/AAS Astrodynamics Specialist Conference, Toronto, ON, Canada, 2-5 August 2010; p. 7652.

20. Nobari, N.A.; Misra, A.K. A hybrid attitude controller consisting of electromagnetic torque rods and an active fluid ring. Acta Astronaut. 2014, 94, 470-479. [CrossRef]

21. Tayebi, J.; Soleymani, A. A comparative study of CMG and FMC actuators for nano satellite attitude control system-pyramidal configuration. In Proceedings of the 2015 7th International Conference on Recent Advances in Space Technologies (RAST), Istanbul, Turkey, 16-19 June 2015; pp. 359-365.

22. Akbaritabar, S.; Esmaelzadeh, R.; Zardashti, R. Comparing fluid ring and CMG servomechanisms for active control of rigid satellites. Aircr. Eng. Aerosp. Technol. 2018, 90, 896-905. [CrossRef]

23. Maschke, B.M.; van der Schaft, A.J. Port-controlled Hamiltonian systems: Modelling origins and systemtheoretic properties. In Nonlinear Control Systems Design 1992; Elsevier: Amsterdam, The Netherlands, 1993; pp. 359-365.

24. Ortega, R.; Van Der Schaft, A.; Maschke, B.; Escobar, G. Interconnection and damping assignment passivity-based control of port-controlled Hamiltonian systems. Automatica 2002, 38, 585-596. [CrossRef]

25. Xi, Z.; Cheng, D.; Lu, Q.; Mei, S. Nonlinear decentralized controller design for multimachine power systems using Hamiltonian function method. Automatica 2002, 38, 527-534. [CrossRef]

26. Xi, Z. Adaptive stabilization of generalized Hamiltonian systems with dissipation and its applications to power systems. Int. J. Syst. Sci. 2002, 33, 839-846. [CrossRef]

27. Wassink, M.; Carloni, R.; Stramigioli, S. Port-Hamiltonian analysis of a novel robotic finger concept for minimal actuation variable impedance grasping. In Proceedings of the 2010 IEEE International Conference on Robotics and Automation, Anchorage, AK, USA, 3-7 May 2010; pp. 771-776.

28. Yu, H.; Yu, J.; Liu, J.; Song, Q. Nonlinear control of induction motors based on state error PCH and energy-shaping principle. Nonlinear Dyn. 2013, 72, 49-59. [CrossRef]

29. Chi, J.; Yu, H.; Yu, J. Hybrid tracking control of 2-DOF SCARA robot via port-controlled hamiltonian and backstepping. IEEE Access 2018, 6, 17354-17360. [CrossRef]

30. Zhao, B.; Yu, H.; Yu, J.; Liu, X.; Wu, H. Port-controlled Hamiltonian and sliding mode control of gantry robot based on induction motor drives. IEEE Access 2018, 6, 43840-43849. [CrossRef]

31. Beattie, C.A.; Mehrmann, V.; Van Dooren, P. Robust port-Hamiltonian representations of passive systems. Automatica 2019, 100, 182-186. [CrossRef]

32. Battin, R.H. An Introduction to the Mathematics and Methods of Astrodynamics, Revised ed.; American Institute of Aeronautics and Astronautics: Reston, VA, USA, 1999.

33. Junkins, J.L.; Schaub, H. Analytical Mechanics of Space Systems; American Institute of Aeronautics and Astronautics: Reston, VA, USA, 2009.

34. Nelson, R.C. Flight Stability and Automatic Control; WCB/McGraw Hill: New York, NY, USA, 1998; Volume 2.

35. Junkins, J.L.; Turner, J.D. Optimal Spacecraft Rotational Maneuvers; Elsevier: Amsterdam, The Netherlands, 2012.

36. Van Der Schaft, A. Port-Hamiltonian systems: An introductory survey. In Proceedings of the International Congress of Mathematicians, Madrid, Spain, 27-30 August 2006; Volume 3, pp. 1339-1365.

37. Duindam, V.; Macchelli, A.; Stramigioli, S.; Bruyninckx, H. Modeling and Control of Complex Physical Systems: The Port-Hamiltonian Approach; Springer Science \& Business Media: Berlin, Germany, 2009.

38. Van Der Schaft, A.; Jeltsema, D. Port-Hamiltonian systems theory: An introductory overview. Found. Trends Syst. Control 2014, 1, 173-378. [CrossRef]

39. Taylor, J.R. Classical Mechanics; University Science Books: Herndon, VA, USA, 2005.

40. Yunus, A.C. Fluid Mechanics: Fundamentals And Applications (Si Units); Tata McGraw Hill Education Private Limited: New York, NY, USA, 2010.

41. Wang, C.; Shi, W.; Wang, X.; Jiang, X.; Yang, Y.; Li, W.; Zhou, L. Optimal design of multistage centrifugal pump based on the combined energy loss model and computational fluid dynamics. Appl. Energy 2017, 187, 10-26. [CrossRef]

42. Shao, C.; Zhao, Y. Numerical study of the dimensionless characteristics and modeling experiment of a molten salt pump that transports viscous fluids. Int. J. Numer. Methods Heat Fluid Flow 2017, 27, 2131-2153. [CrossRef]

43. Freund, R.J.; Wilson, W.J.; Sa, P. Regression Analysis; Elsevier: Amsterdam, The Netherlands, 2006.

44. Landau, L.D.; Lifshitz, E.M. Mechanics: Volume 1; Butterworth-Heinemann: Oxford, UK, 1976.

45. Feynman, R.P.; Leighton, R.B.; Sands, M. The Feynman Lectures on Physics, Vol. I: The New Millennium Edition: Mainly Mechanics, Radiation, and Heat; Basic Books: New York, NY, USA, 2011. 